\title{
Evaluating the Trade-off Between Energy Efficiency and QoE in Wireless Mesh Networks
}

\author{
David Hock*, Vitor Bernardo ${ }^{\dagger}$, Thomas Zinner*, Florian Wamser*, Karin A. Hummel ${ }^{\ddagger}$, Marilia Curado $^{\dagger}$, \\ Rastin Pries*, Torsten Braun ${ }^{\S}$, Phuoc Tran-Gia* \\ *University of Würzburg — Email: \{david.hock,zinner,florian.wamser,pries,trangia\} @informatik.uni-wuerzburg.de \\ ${ }^{\dagger}$ University of Coimbra — Email: \{vmbern,marilia\}@ dei.uc.pt \\ ${ }_{\ddagger}^{\ddagger}$ ETH Zürich — Email: karin.hummel@tik.ee.ethz.ch \\ $\S$ University of Bern — Email: braun@iam.unibe.ch
}

\begin{abstract}
The increasing usage of wireless networks creates new challenges for wireless access providers. On the one hand, providers want to satisfy the user demands but on the other hand, they try to reduce the operational costs by decreasing the energy consumption. In this paper, we evaluate the tradeoff between energy efficiency and quality of experience for a wireless mesh testbed. The results show that by intelligent service control, resources can be better utilized and energy can be saved by reducing the number of active network components. However, care has to be taken because the channel bandwidth varies in wireless networks. In the second part of the paper, we analyze the trade-off between energy efficiency and quality of experience at the end user. The results reveal that a provider's service control measures do not only reduce the operational costs of the network but also bring a second benefit: they help maximize the battery lifetime of the end-user device.

Index Terms - energy efficiency, quality of experience, broadband wireless access, wireless mesh networks
\end{abstract}

\section{INTRODUCTION}

In today's wireless access networks, a huge portion of the operational costs (OPEX) is used for empowering wireless devices. Thus, the primary goal of an Internet Service Provider (ISP) is to reduce the energy consumption without harming the quality of experience (QoE) of the end user as this would result in unsatisfied customers. To solve this trade-off, the ISP has two options, network control and service control.

Using network control, the ISP adds and removes resources based on the current traffic load. For instance, if the traffic load is high during business hours, additional femtocells can be launched in a mobile communication network to cope with the traffic demand. In case of low load, the femtocells can be switched off again. When using service control, the ISP tries to improve the situation in the network by controling the services at the client. This can be achieved by identifying the services currently running in the network, for instance, with deep packet inspection (DPI). If the situation in the network is not satisfactory, the required bandwidth of the services is reduced, for instance, by decreasing the resolution of a video. The impact of service control on QoE for different services was published by Hoßfeld et al. [1]. It is shown that a huge amount of bandwidth can be saved in wired networks with only a minor QoE degradation. Thus, network resources can be switched off, the energy can be saved, while the impact on the QoE is negligible.
In wireless networks, service control has to consider two issues. On the one hand, the available bandwidth varies, depending on fading and other attenuation effects, which exacerbates to guarantee a fixed bandwidth. On the other hand, interference problems may occur when adding more wireless resources.

In this paper, we investigate service control in wireless networks at the access network and at the end user device. We discuss when it is beneficial to reduce the energy consumption and when to add resources to satisfy the user's expectations in terms of QoE for two major sample applications, web access and video streaming.

For the access network, we demonstrate how service control can adapt the number of resources to work energy-efficient, i.e., providing the required $\mathrm{QoE}$ while reducing the energy consumption. Hereby, we also argue that there are limitations of simple network control as adding more wireless resources may lead to more interferences and to a decrease of network performance. The results are based on measurements in an IEEE 802.11 wireless mesh testbed. We illustrate the trade-off for web access in two different scenarios, one when the user needs only a single wireless hop to connect to the Internet and the other scenario when the user requires two wireless hops to the Internet gateway.

At the edge of the network, we analyze the trade-off between energy efficiency and QoE at the end user device. Therefore, we look at the end user energy requirements needed to support high-definition (HD) video streaming on different quality levels.

The remainder of this paper is organized as follows. In Section II we briefly explain the background of this work and provide an overview of related work. Section III addresses energy efficiency in the access network, and the topic of Section IV is energy efficiency at the edge of the network, i.e., at the end user device. Section $\mathrm{V}$ concludes the paper and summarizes the findings.

\section{BACKGROUND AND RELATED WORK}

Energy efficiency in wireless mesh networks can be achieved by local and distributed concepts as summarized, e.g., in [2]. Local concepts are switching network interfaces on and off, adapting transmission ranges, modulation and 
coding schemes, and selecting wireless links consuming less energy while providing sufficient quality of network services. Distributed concepts include decreasing the overall capacity of the network by switching components on and off (i.e., avoiding over-provisioning), decreasing the number of active devices while increasing transmission power, or using other compensation methods for service degradation ranging from text compression to delay tolerant networking (DTN) protocols that tolerate temporary loss of connectivity. Awareness of the current situation or context can help to power up exactly the networking components necessary to provide the required network services as done for estimating wireless network conditions [3]. Our work contributes to enhancing service control by reducing active network components if the resulting QoE is sufficient.

In the context of video transmission, the trade-off between the experienced video quality and the energy consumed has to be addressed. In [4], depending on the video quality, the sleep time of the wireless network interface is adapted. $\mathrm{Li}$ et al. [5] studied energy-efficient video transmission over wireless links by controlling parameters associated with the physical and link layers. Simulation results show energy savings of nearly $40 \%$ for a Code Division Multiple Access (CDMA) system supporting six users. Other simulation-based approaches have investigated energy-efficient video transmission based on scalable video coding using content-aware rate control [6] and cooperative video transmission with end-toend statistical quality of service provisioning [7]. Yuan et al. [8] used cross-layer techniques to improve the quality of multimedia applications while minimizing battery energy consumption. The energy consumption is assessed in a testbed using a digital oscilloscope. The Phoenix system is another cross-layer based approach to minimize network overhead for video stream transmission by applying scalable video coding and adaptive error control based on exchanging signaling and control messages [9]. Error resilience and concealment techniques at the source coding level, and transmission power management at the physical layer have been combined in [10] to minimize energy consumption of video transmission over wireless networks. Other works have studied the impact of transmitting video on the QoE perceived by the end user under different network conditions, but without any consideration of energy consumption [11], [12].

\section{QOE AND ENERGY EFFICIENCY IN THE ACCESS NETWORK}

First, we investigate the trade-off between QoE and energy efficiency in the access network, i.e., in the nodes that provide access to the Internet for the end user. As a practical example, we look at web browsing over wireless mesh networks. We also made similar evaluations for video streaming traffic as addressed in [1] and it yield similar insights. Due to lack of space, in this section we address only web traffic and video streaming traffic is addressed later in Section IV as an example for end user device energy efficiency. First, we describe the methodology and the components used for the evaluation. Then, we present our evaluation results.

\section{A. Methodology}

In the following, we first briefly address web traffic as the application considered in this work. Then, we provide information about our energy efficiency metric. Finally, we show the two different wireless access network scenarios studied in our evaluation.

1) Application under study: In the access network, we consider web traffic as an example application. We are mainly interested in the number of web users that can be supported on a given network capacity. Therefore, we use a QoE mapping function based on the bandwidth $b w$ of the application by providing an adequate reference value for the minimum necessary bandwidth to reach the best possible QoE.

According to Fiedler et. al [13], the QoE of a web user corresponds to the maximum mean opinion score (MOS) 5, if the weighted session time is no longer than about $0.5 \mathrm{~s}$. If we assume a web page size of $500 \mathrm{kByte}$ (which corresponds to the $90 \%$ percentile of all web page sizes [14]), a bandwidth of $b w=8 \mathrm{Mbps}$ is needed to download the web page in $0.5 \mathrm{~s}$. Therefore, we assume $Q o E_{w e b}(b w)$ to be 5 for $b w \geq 8 \mathrm{Mbps}$.

For smaller values of $b w$ we use a mapping function based on the one proposed by Hoßfeld, et al. [1]:

$$
Q o E_{w e b}(b w)=\max \left\{1,5+1.5 \ln \left(\frac{b w}{8}\right)\right\} \text {. }
$$

2) Energy efficiency metrics: To measure the energy efficiency of a certain scenario, we consider the power consumption of single nodes in a wireless mesh network. As reference value, we measure with a power meter the power consumption of a Saxnet Meshnode III as these are the nodes used in the wireless mesh testbed at the University of Wuerzburg. Equipped with four IEEE 802.11 interfaces, these nodes are able to use up to four different channels in parallel.

Table I shows the measured values. The largest amount of the total power consumption of a node is the base power needed for the system to run. Each additional wireless interface increases the power consumption. Note that the power increase is not equal for the different interfaces but increasing from 0.7 Watts for the first interface to 2.1 Watts for the last interface. During our measurements, we recognized that not all four wireless interfaces of the node can be fully loaded at the same time as in this case, the CPU turned out to be the bottleneck. Therefore, we can only provide adequate values for the power consumption of up to three interfaces. Thus, in

TABLE I

MEASURED POWER CONSUMPTION OF A WIRELESS MESH NODE.

\begin{tabular}{cc}
\hline State description & Power consumption \\
\hline Idle, all wireless interfaces switched off & 13.3 Watts \\
One wireless interface in use (full load) & 14.0 Watts \\
Two wireless interfaces in use (full load) & 15.7 Watts \\
Three wireless interfaces in use (full load) & 17.8 Watts \\
\hline
\end{tabular}




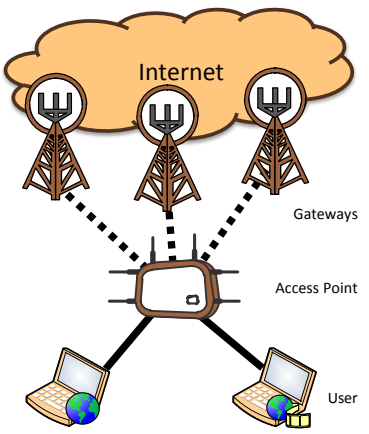

(a) Single hop scenario.

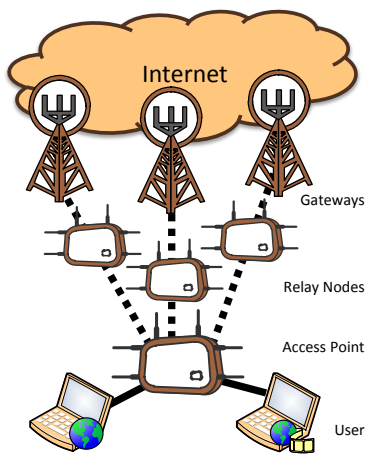

(b) Multi hop scenario.
Fig. 1. Scenarios under study.

all scenarios considered in this paper we limit the number of interfaces a node uses at the same time to three.

3) Scenarios: We consider two different scenarios, as depicted in Figure 1. The first scenario is a single hop scenario as illustrated in Figure 1(a). One mesh node is configured as an access point and allows users to connect to the wireless mesh network. Up to three mesh nodes are configured as mesh gateways providing Internet access. In our scenario, the end users are connected to the access point via Ethernet and the gateways have a fixed access, e.g. via DSL, to the Internet. The wireless transmission in the scenario is only a single hop from the access point to the gateways.

The second scenario represents a situation where it is not possible to connect the access point directly to the gateways via a single wireless hop. Therefore, additional mesh nodes are used as relay nodes that receive the traffic from the access point and forward it to the gateways, as shown in Figure 1(b).

The performance evaluation in Section III-B shows that the conditions between the two considered scenarios differ significantly and that this has to be taken into account when a trade-off between energy efficiency and QoE has to be found.

\section{B. Performance Evaluation}

We briefly show measurement results of our wireless mesh testbed used as a reference for further evaluations. Then, we discuss the trade-off between energy efficiency and QoE.

1) Bandwidth analysis in a wireless mesh testbed: In fixed local area networks, the maximum transmission bandwidth between two directly connected hops can normally be expected to be rather constant. In general, this does not hold in wireless environments. In a WiFi network based on the IEEE 802.11 standard, the bandwidth between two hops is subject to variations due to fading, multipath propagation, modulation, etc. This effect is even increased if the signals of several wireless nodes interfere and thereby cause retransmissions leading to a temporary lower total bandwidth.

For this paper, we did a reference measurement at the wireless mesh testbed at the University of Wuerzburg consisting of four Saxnet Meshnode III as well as several additional wireless nodes configured as gateways. Table II summarizes the mean bandwidth values and the $5 \%$ percentiles of the bandwidth values in Mbps for all considered mesh scenarios.
TABLE II

TRANSMISSION BANDWIDTHS FOR DIFFERENT POSSIBLE MESH SCENARIOS IN MBPS.

\begin{tabular}{cccc}
\hline scenario & mean & 5\% percentile & relative gap \\
\hline Single hop, \#Gateways: 1 & 22.1268 & 19.9000 & $10.06 \%$ \\
Single hop, \#Gateways: 2 & 21.8524 & 19.9000 & $8.93 \%$ \\
Single hop, \#Gateways: 3 & 21.1413 & 19.9000 & $5.87 \%$ \\
\hline Multi hop, \#Gateways: 1 & 19.1520 & 16.4000 & $14.37 \%$ \\
Multi hop, \#Gateways: 2 & 17.8784 & 13.3000 & $25.61 \%$ \\
Multi hop, \#Gateways: 3 & 14.5837 & 8.4500 & $42.06 \%$ \\
\hline
\end{tabular}

The relative gap presents the relative decrease of bandwidth between the mean and the $5 \%$ percentile. We considered the single hop (SH) and multi hop $(\mathrm{MH})$ case for one, two, or three activated gateways at the same time. We measured the maximum possible TCP throughput on all links used in the corresponding scenario. For instance, in the single hop scenario with two gateways the links between the access point and two different gateways were utilized in parallel.

The values reveal several interesting facts. In the single hop scenarios, the mean bandwidth is at a high base level and only slightly reduced by the presence of additional gateways. However, in the single hop scenario with one gateway, in $5 \%$ of the cases the bandwidth might decrease by up to $10.06 \%$ in our mesh testbed.

In the multi hop case, there are two main effects. On the one hand, there is the effect of interference. An increase of the number of gateways from 1 to 3 drastically reduces the average bandwidth per gateway in our reference testbed. Note that this is dependent on the actual placement of the nodes however it means that doubling the number of gateways does not necessarily lead to a doubling of the available resources in terms of bandwidth. On the other hand, the increase of gateways and the interference leads to a much higher variation of the bandwidth, and increases the relative gap to up to $42 \%$ in case of three gateways. In other words, in $5 \%$ of the measured cases, the bandwidth was less than $60 \%$ of the average bandwidth. As a consequence, if a wireless network operator uses the average bandwidth in such a wireless network as reference value for network provisioning, in $5 \%$ of the time the actual bandwidth would experience extreme deviations to the average value and thus to the expected QoE. In the following, this effect is quantified using the example of web traffic.

2) Trade-off between energy efficiency and QoE for web traffic: The goal of our analysis is twofold. On the one hand, we want to find out how the average QoE that can be offered to each single user depends on the total number of supported users $N$ in the network and the number of activated gateways. On the other hand, within the scope of energy efficiency, we want to know how much power in Watt is necessary to support a certain number of users.

The equation given in Section III-A allows for a direct mapping of a user's bandwidth $b w$ to the perceived quality $Q o E_{w e b}(b w)$. Evidently, if in wireless networks the bandwidth is subject to variations, the resulting QoE will also retain this behavior. This has to be considered in the evaluations and is illustrated in Figure 2. 


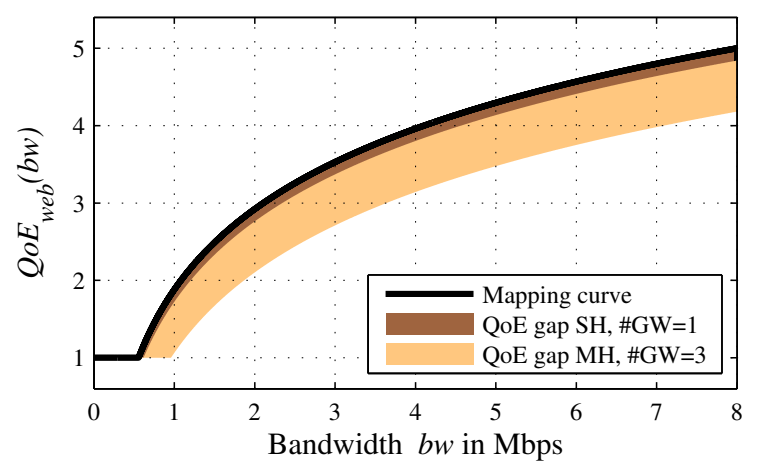

Fig. 2. Web traffic model.

If we imply that the bandwidth is equally spread among all users, obviously the bandwidth gap is also spread equally among the users. Due to the logarithmic nature of the mapping, if the bandwidth $b w$ is decreased multiplicatively to $b w \cdot(1-g a p)$, the quality is decreased additively by a "QoE gap" $1.5 \ln (1-$ gap $)$.

Figure 2 shows the QoE gap for two different scenarios, single hop with one gateway and multi hop with three gateways. While in the first case, with bandwidth gap $10.06 \%$, the QoE gap is only 0.1590 , in the second case, with $42.06 \%$, the QoE gap is 0.8186 . This means that in $5 \%$ of the time, the MOS of a given user can be by more than 0.8 worse than the average MOS of that user. This might be acceptable for a base MOS value of 5 reduced to about 4.2, but it is not feasible for a base MOS value of 3 reduced to about 2.2. A network operator should consider this during the service provisioning.

Based on the previous observation, we now analyze how the average QoE of the users and the energy consumption depends on the total number of supported users in the network.

The first question to answer is what average QoE can be provided to each single user depending on the number of users and gateways in the network.

Analogously to [1] (Section V), we assume that the available bandwidth of a gateway is equally distributed among all users connected to the gateway. Let $C$ be the gateway capacity in Mbps and $N$ the number of users. Then, the bandwidth of each user on this gateway equals $b w=\frac{C}{N}$ and accordingly all users on the gateway have the same QoE (i.e., $Q o E_{w e b}(b w)$ ). Thus, the average QoE for a given $C$ and $N$ can simply be calculated as $Q_{o} E_{w e b}\left(\frac{C}{N}\right)$.

Based on this metric, Figure 3 shows the average QoE of the users depending on the number of users in the network and the number of gateways for the single hop and the multi hop scenario. The bold lines represent the case when the average bandwidth in each scenario is regarded, the thin line represents the $5 \%$ percentile case.

As long as the fraction of bandwidth each user obtains is higher than $8 \mathrm{Mbps}$, the average MOS of the users is 5 . For the single hop, this is the case for 2 users in the one gateway scenario with an average bandwidth of $22.13 \mathrm{Mbps}$. The decrease of the average QoE by 1 allows for the admission of about twice the number of users in the network, e.g. 5 users with MOS 4 in the single hop scenario with one gateway.

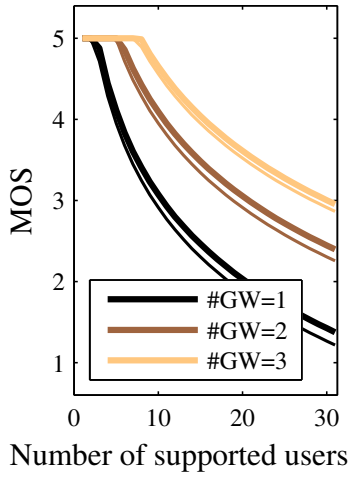

(a) Single hop scenario.

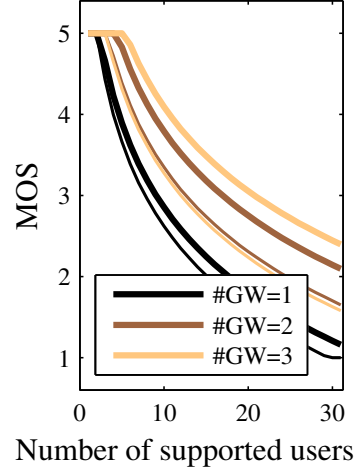

(b) Multi hop scenario.
Fig. 3. Providable MOS depending on the number of web users for different number of gateways.

The activation of additional gateways increases the available resources to $g \cdot C_{g}$, where $g$ is the number of gateways and $C_{g}$ is the capacity of a gateway in the $g$ gateway scenario.

There are additional important effects caused by the wireless environment. A decrease of the average QoE does not only increase the number of supported users in the network but also the number of users in the QoE gap. For instance, in the single hop scenario with two gateways, on average 5 users with $Q o E_{w e b}=5$ can be supported. However, if the $5 \%$ percentile is regarded, only 4 users can be assigned with this value. This gap of one user is increased to two users, when $Q o E_{w e b}=3$ is considered. For the network operator, this means that in case the bandwidth temporarily drops to the lower value, they can either reduce the QoE of all users accordingly or keep the QoE of most users at the same level and block a number of users equal to the size of the gap. To proactively avoid such situations, the provider might prefer to activate an additional gateway directly to increase both average and percentile bandwidth.

Figure 3(b) shows the same dependency of QoE and number of users for the multi hop scenarios. One can directly see the impact of two main wireless effects. First, due to the interference of the different gateways, the increase in the number of supported users by the activation of additional gateways is lesser than the increase in the single hop case. Second, the QoE gap is much higher leading even to the fact that $2 \cdot C_{2}=2 \cdot 13.3=26.6>25.35=3 \cdot 8.45=3 \cdot C_{3}$ if the $5 \%$ percentiles are regarded. With other words, in the multi hop case, in $5 \%$ of the time, the capacity in the network with two active gateways might be higher than the one with three active gateways. This should be taken into account by the network providers.

Now, we investigate the necessary power consumption in terms of Watt to allow for a certain number of users. Therefore, based on the reference values provided in Table I, we estimate the power consumption of different scenarios including different numbers of nodes and interfaces.

Figure 4 shows for different scenarios and for different average QoE levels, at which user count the next gateway should be activated. Again, a large gap between the average 


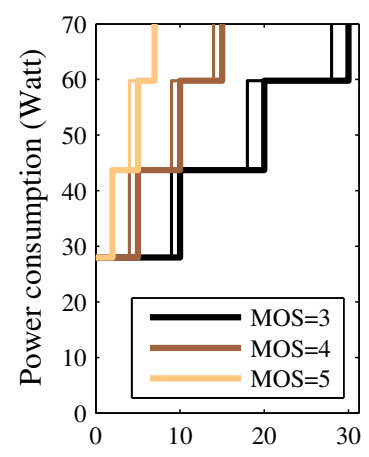

Number of supported users

(a) Single hop scenario.

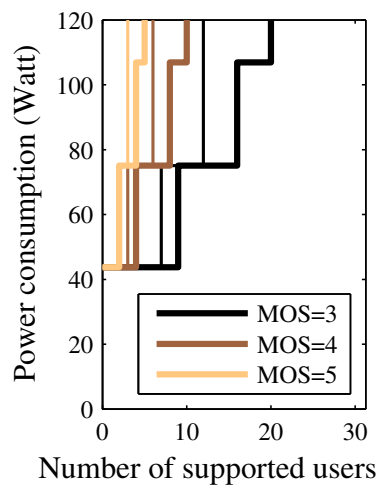

(b) Multi hop scenario.
Fig. 4. Necessary costs to support a certain number of web users.

supported number of users and the $5 \%$ percentile can be seen, especially for the multi hop scenarios. In particular, as mentioned before, when considering the $5 \%$ percentile it might be better even not to activate the third gateway but to start admission control as soon as no more users can be supported on the desired QoE level with two gateways.

In the single hop scenarios as visualized in Figure 4(a), the connection of the users to the Internet via one gateway leads to a total power consumption of 28.0 Watts corresponding to two mesh nodes with one active interface each (access point and gateway 1). The second gateway increases the consumption by 15.7 Watts as one additional node (14.0 Watts) and the second interface at the access point (1.7 Watts) are activated. Finally, the third gateway requires additional 16.1 Watts consisting of the third gateway (14.0 Watts) and the third interface of the access point (2.1 Watts). Thus, due to the large amount of system power consumption compared to the little wireless interface consumption, the costs raise almost linearly with the number of gateways.

The same holds for the multi hop scenario. However, here with each additional activated gateway, not only the gateway node has to be started, but also one new relay node with two wireless interfaces. This leads to an additional increase per step of 15.7 Watts.

Before, we recognized that doubling the gateway count does in general not double the available bandwidth due to interference problems. As the costs increase linearly to the gateway count, this can be mapped to the following: paying twice the price does not mean to be able to satisfy double the number of users.

\section{ENERgy EFFICIENCY AT THE EdGE}

In order to integrate the end user device into energy efficiency considerations, this section presents an experimental assessment of the energy requirements of an end user device. To exemplify in a concrete example, the evaluation was conducted assessing the power consumption needed to support a high-definition (HD) Internet streaming in a wireless access scenario, while keeping the end user QoE on a desired level. In this measurement scenario, the entire access network including mesh access point and mesh gateway is reduced to a single

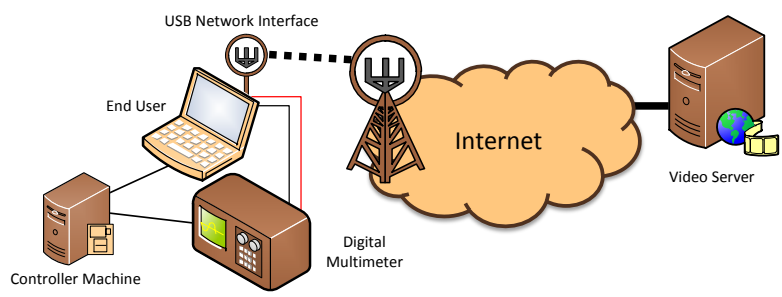

Fig. 5. Measurement methodology.

entity. Therefore, the only wireless link is the hop between the end user and the access point. Evidently, as the scope is on the user device here, the basic results hold also for a scenario with more complex access networks as long as the necessary bandwidth for the video can be guaranteed in the access network.

\section{A. Methodology}

The employed energy measurement methodology [15] depicted in Figure 5 has two main components: the energy measurement, and the video traffic generation / evaluation. The energy measurement is performed using an external USB network interface, which enables the system to be used with different wireless technologies (e.g., WiFi, GPRS). The measurement configuration includes the end user device, a highprecision digital multimeter, and a "controller machine" (for remote management of the multimeter), which allows accurate and repeatable tests.

The video traffic generation was performed using the Evalvid framework [16], since this framework is able to reconstruct the received video, even when some frames have been lost. Evalvid can report QoS metrics, such as packet loss and one-way delay, and QoE metrics, such as the MOS.

\section{B. Experimental Results}

The experimental results for the end device were obtained using the University of Coimbra testbed facilities [15]. To evaluate high-definition video streaming, we obtained a highdefinition (1920x1080 pixel) movie from the TUM Multi Format Test Set [17]. The select video named "ski2" has a duration of 12 seconds and shows a camera record of a skiing person. The tests were conducted compressing the "ski2" lossless quality sequence employing five different bit rates, ranging from $2 \mathrm{Mbps}$ to $10 \mathrm{Mbps}$, and using a MPEG-4 codec. The results presented include 10 runs for each distinct setup with a confidence interval of $95 \%$.

Figure 6 shows the average power consumption in Watts consumed by the end user's network interface when receiving the desired video (y-axis on the right), and the corresponding QoE, expressed by the MOS metric (y-axis on the left). The $\mathrm{X}$-axis represents the different video bit rates tested.

The energy needed to receive the compressed "ski2" video increases with the bit rate. When assessing the perceived end user QoE, it is noticeable that only a slightly quality improvement is achieved, even when using a high bit rate movie (i.e., $10 \mathrm{Mbps}$ ). In fact, this behavior depends on the used video. For instance, due to the encoding process, a video with less similarities between consecutive frames requires a 


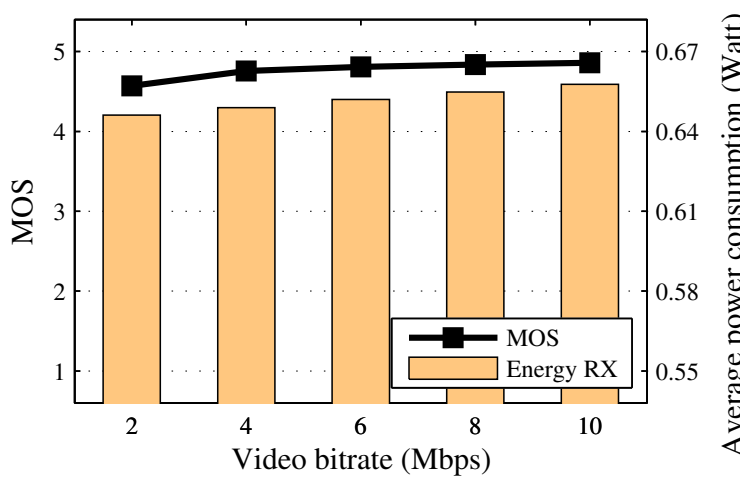

Fig. 6. HD video streaming average power consumption and MOS

higher bit rate to reach the same quality as a more static video. In this case, the trade-off between power consumption and the attained QoE is reasonable with the $2 \mathrm{Mbps}$ stream with MOS $=4.57$. The MOS is close to the optimal value when employing the $4 \mathrm{Mbps}$ stream (MOS = 4.76). The $10 \mathrm{Mbps}$ bit rate movie is only capable to reach a MOS of 4.86 , which does not represent a considerable quality improvement for the end user when compared with the previous sequences.

Analyzing the obtained results, it is possible to conclude that service control techniques, applied to video streaming quality, do not only bring benefits to the operator, as shown in Section III. They also allow to reduce the energy consumption at the end users and thus help to improve the device battery lifetime.

\section{CONCLUSION}

In this paper, we have shown the trade-off between energy efficiency and QoE in various scenarios both from the network and the end user perspective. In fixed networks, service control is a good option to reduce the required bandwidth and thus, to reduce the energy consumption by switching off unnecessary components. We presented that safety margins for service control have to be introduced because the available bandwidth in wireless networks varies due to different distortions and interferences.

Scenarios with web users illustrate that wireless network distortions can easily lead to an unacceptable QoE in particular in multi hop scenarios. Adding more resources, for instance, by switching on more Internet gateways, helps to increase the user satisfaction up to a certain point but also comes along with an increased energy consumption. Our results have shown that doubling the number of Internet gateways does not double the capacity in terms of bandwidth. On the contrary, it can also happen that the additional resources are completely wasted due to interference problems.

Concerning the edge of the network, we investigated the end user device energy requirements needed to support highdefinition Internet video streaming. We showed that the energy consumption might be significantly reduced by allowing for a slightly reduction of the transmitted video quality.

Summarizing our findings, we propose to use service control in a high load scenario with a safety margin due to bandwidth variations. This helps to reduce the energy consumption both in the network and at the end user.

\section{ACKNOWLEDGMENTS}

This work was partially supported by the COST (European Cooperation in Science and Technology) framework, under Action IC0804 and by the EC under the FP7 Marie Curie IEF program contract PIEF-GA-2010-276336 MOVE-R.

The second author was supported by the Portuguese Foundation for Science and Technology (FCT) through a Doctoral Grant (SFRH/BD/66181/2009).

\section{REFERENCES}

[1] T. Hoßfeld, M. Fiedler, and T. Zinner, "The QoE Provisioning-DeliveryHysteresis and Its Importance for Service Provisioning in the Future Internet," in Proceedings of the 7th Conference on Next Generation Internet Networks (NGI), Kaiserslautern, Germany, June 2011.

[2] Y. Al-Hazmi, H. de Meer, K. Hummel, H. Meyer, M. Meo, and D. Remondo, "Energy-efficient Wireless Mesh Infrastructures," IEEE Network, vol. 25, no. 2, pp. 32 -38, March-April 2011.

[3] A. Rahmati and L. Zhong, "Context-for-wireless: context-sensitive energy-efficient wireless data transfer,' in MobiSys '07: Proceedings of the 5th international conference on Mobile systems, applications and services, 2007, pp. 165-178.

[4] M. Csernai and A. Gulyas, "Wireless adapter sleep scheduling based on video qoe: How to improve battery life when watching streaming video?" in 20th International Conference on Computer Communications and Networks (ICCCN), 2011, pp. $1-6$.

[5] Y. Li, M. Reisslein, and C. Chakrabarti, "Energy-efficient video transmission over a wireless link," Vehicular Technology, IEEE Transactions on, vol. 58, no. 3, pp. 1229 -1244, March 2009.

[6] S. Lee, J. Koo, and K. Chung, "Content-aware rate control scheme to improve the energy efficiency for mobile iptv," in Consumer Electronics (ICCE), 2010 Digest of Technical Papers International Conference on, January 2010, pp. $445-446$.

[7] A. A. Khalek and Z. Dawy, "Energy-efficient cooperative video distribution with statistical qos provisions over wireless networks," IEEE Transactions on Mobile Computing, vol. 99, no. PrePrints, 2011.

[8] W. Yuan, K. Nahrstedt, S. Adve, D. Jones, and R. Kravets, "Grace-1: cross-layer adaptation for multimedia quality and battery energy," Mobile Computing, IEEE Transactions on, vol. 5, no. 7, July 2006.

[9] J. Huusko, J. Vehkaperä, P. Amon, C. Lamy-Bergot, G. Panza, J. Peltola, and M. G. Martini, "Cross-layer architecture for scalable video transmission in wireless network," Image Commun., vol. 22, March 2007.

[10] Y. Eisenberg, C. Luna, T. Pappas, R. Berry, and A. Katsaggelos, "Joint source coding and transmission power management for energy efficient wireless video communications," Circuits and Systems for Video Technology, IEEE Transactions on, vol. 12, no. 6, June 2002.

[11] K. De Moor, I. Ketyko, W. Joseph, T. Deryckere, L. De Marez, L. Martens, and G. Verleye, "Proposed framework for evaluating quality of experience in a mobile, testbed-oriented living lab setting," Mobile Networks and Applications, vol. 15, pp. 378-391, 2010.

[12] V. Bernardo, K. Pentikousis, J. Pinola, E. Piri, and M. Curado, "Multiclient video streaming over wirelessman-ofdma," in Proceedings of the 4th ACM workshop on Performance monitoring and measurement of heterogeneous wireless and wired networks, ser. PM2HW2N '09. New York, NY, USA: ACM, 2009, pp. 46-53.

[13] M. Fiedler and T. Hoßfeld, "Quality of Experience-Related Differential Equations and Provisioning-Delivery Hysteresis," in to 21st ITC Specialist Seminar on Multimedia Applications - Traffic, Performance and QoE, Phoenix Seagaia Resort, Miyazaki, Japan, March 2010.

[14] P. Ameigeiras, J. Ramos-Munoz, J. Navarro-Ortiz, P. Mogensen, and J. Lopez-Soler, "Qoe oriented cross-layer design of a resource allocation algorithm in beyond 3g systems," Computer Communications, vol. 33, no. $5,2010$.

[15] V. Bernardo and M. Curado, "A methodology for assessing video transmission energy consumption and quality," in International Workshop on Novel approaches to Energy Measurement and Evaluation in Wireless Networks (ICC'12 WS - NovaEnEv), Ottawa, Canada, June 2012.

[16] J. Klaue, B. Rathke, and A. Wolisz, "EvalVid - A Framework for Video Transmission and Quality Evaluation," in Proceedings of the 13th Conference on Modelling Techniques and Tools for Computer Performance Evaluation, ser. Lecture Notes in Computer Science. Springer, 2003.

[17] Technische Universität München, Institute for Data Processing. (2011) TUM LDV Multi Format Test Set. 